\title{
Linear non-causal optimal control of an attenuator type wave energy converter M4
}

DOI:

10.1109/TSTE.2019.2922782

\section{Document Version}

Accepted author manuscript

Link to publication record in Manchester Research Explorer

\section{Citation for published version (APA):}

Liao, Z., Gai, N., Stansby, P., \& Li, G. (2019). Linear non-causal optimal control of an attenuator type wave energy converter M4. IEEE Transactions on Sustainable Energy. https://doi.org/10.1109/TSTE.2019.2922782

\section{Published in:}

IEEE Transactions on Sustainable Energy

\section{Citing this paper}

Please note that where the full-text provided on Manchester Research Explorer is the Author Accepted Manuscript or Proof version this may differ from the final Published version. If citing, it is advised that you check and use the publisher's definitive version.

\section{General rights}

Copyright and moral rights for the publications made accessible in the Research Explorer are retained by the authors and/or other copyright owners and it is a condition of accessing publications that users recognise and abide by the legal requirements associated with these rights.

\section{Takedown policy}

If you believe that this document breaches copyright please refer to the University of Manchester's Takedown Procedures [http://man.ac.uk/04Y6Bo] or contact uml.scholarlycommunications@manchester.ac.uk providing relevant details, so we can investigate your claim.

\section{OPEN ACCESS}




\title{
Linear non-causal optimal control of an attenuator type wave energy converter M4
}

\author{
Zhijing Liao, Nian Gai, Peter Stansby, Guang Li Member, IEEE
}

\begin{abstract}
Ocean waves provide a promising and abundant renewable energy resource. One reason wave energy technology is still not mature enough for commercialization is the high unit cost of generated electricity. This needs to be improved by a combination of device and associated controller design. A multi-float and multi-mode-motion WEC (M-WEC) enables much higher energy conversion compared with a single-float, single-mode WEC (S-WEC); however, the added complexity in dynamics of a M-WEC makes the corresponding controller design more challenging. While the majority of current WEC control research has been based on the control of S-WECs it has shown that control can significantly improve energy conversion. This paper aims to design a linear non-causal optimal controller for a M-WEC to demonstrate that this improvement also applies to more complex WEC systems. We choose a multibody attenuator type M-WEC called M4 as a case study for which the desirable feature of predominantly linear dynamics has been demonstrated. This means that a linear controller can be designed based on a linear hydrodynamic model without introducing an intractable computational burden for real-time controller implementation. Numerical results show that the linear non-causal optimal controller can significantly improve the power capture of M4 over a broad range of peak spectral wave periods by $40 \%$ to $100 \%$.
\end{abstract}

Index Terms-Wave energy, Optimal control, Non-causal control, Wave prediction.

\section{INTRODUCTION}

Ocean waves have abundant renewable energy which can be harvested by wave energy converters (WECs). However, current wave energy technologies are not yet mature with a relatively high unit cost of the generated electricity compared to other types of renewable energy generation, specifically wind and solar energy. The energy conversion of WECs may be improved by innovative design configuration and associated control strategies. Multi-float and multi-mode-motion WECs (M-WECs) enable much improved energy conversion over single-float and single-motion WECs (S-WECs), e.g. [1], [2]. It has also long been recognized that control plays an important role in significantly improving the energy conversion of WECs [3]. Early model-free control methods for WECs, such as latching control and declutch control, are based on the impedance matching principle [3]. More recent studies show that advanced model-based control such as optimal

Zhijing Liao (z.liao@qmul.ac.uk), Nian Gai (n,gai@qmul.ac.uk) and Guang Li (g.li@qmul.ac.uk) are with the School of Engineering and Material Science, Queen Mary University of London, London, E1 4NS, UK.

Peter Stansby (p.k.stansby@manchester.ac.uk) is with the School of Mechanical, Aerospace and Civil Engineering, University of Manchester, Manchester, M13 9PL, UK.

Corresponding author: Guang Li (g.li@qmul.ac.uk) control [4], model predictive control [5], [6], pseudospectral control [7], [8] have much greater potential to improve WEC performance. The efficacies of these advanced control strategies however rely on the fidelity of the WEC dynamic model. Due to potential high computational demands resulting from high-order dynamic models, their efficacies have been mainly demonstrated for S-WECs, with single body heaving point absorbers being particularly popular. The control of MWECs has not been investigated to our knowledge probably because complex dynamic models and complex controller design combine to become analytically and computationally prohibitive.

This is however the aim of this paper where we present a design case study for a linear non-causal optimal controller for a M-WEC which is reconfigurable in terms of the number of floats, known as M4 [1], [2]. This is essentially a rafttype attenuator device which absorbs energy from the relative pitch motion of floats connected by beams at a hinge. There are three rows of floats with a single bow float attached to a mooring buoy [2]; the floats increase in size downwave and the device heads naturally into the wave direction. In its simplest form there is one mid float connected by a beam to the bow float and a stern float connected by a beam to a hinge above the mid float at which mechanical damping absorbs wave energy [1]. The M4 design has relatively high capture width ratio (CWR) compared to other designs and larger multi-float systems have capacities comparable to offshore wind for certain sites [2], [9]. An important attraction of M4 is that it has inherent linear dynamics in operational conditions shown by comparing laboratory measurements with linear diffraction modelling for the original 3-float version in the frequency domain [10], [11] and the time domain using the Cummins method [2]. This has now also been shown for a 6-float version [9] and up to 8 floats have been modelled [2]. The linear dynamics enable the development of a concise control-oriented model using model order reduction techniques. Based on this reduced-order linear model for M4, a non-causal optimal controller can be developed for its realtime implementation. For this study we use the original 3 float version since this is sufficient to provide proof of concept. The prediction of incoming waves is assumed to be available from the existing prediction techniques, e.g. the deterministic sea wave prediction (DSWP) algorithm [12]. This wave prediction information can be explicitly incorporated into the non-causal optimal controller design and plays a key role in improving the performance of M4 as demonstrated in the simulations of the paper.

The rest of this paper is organized as follows. In Section 


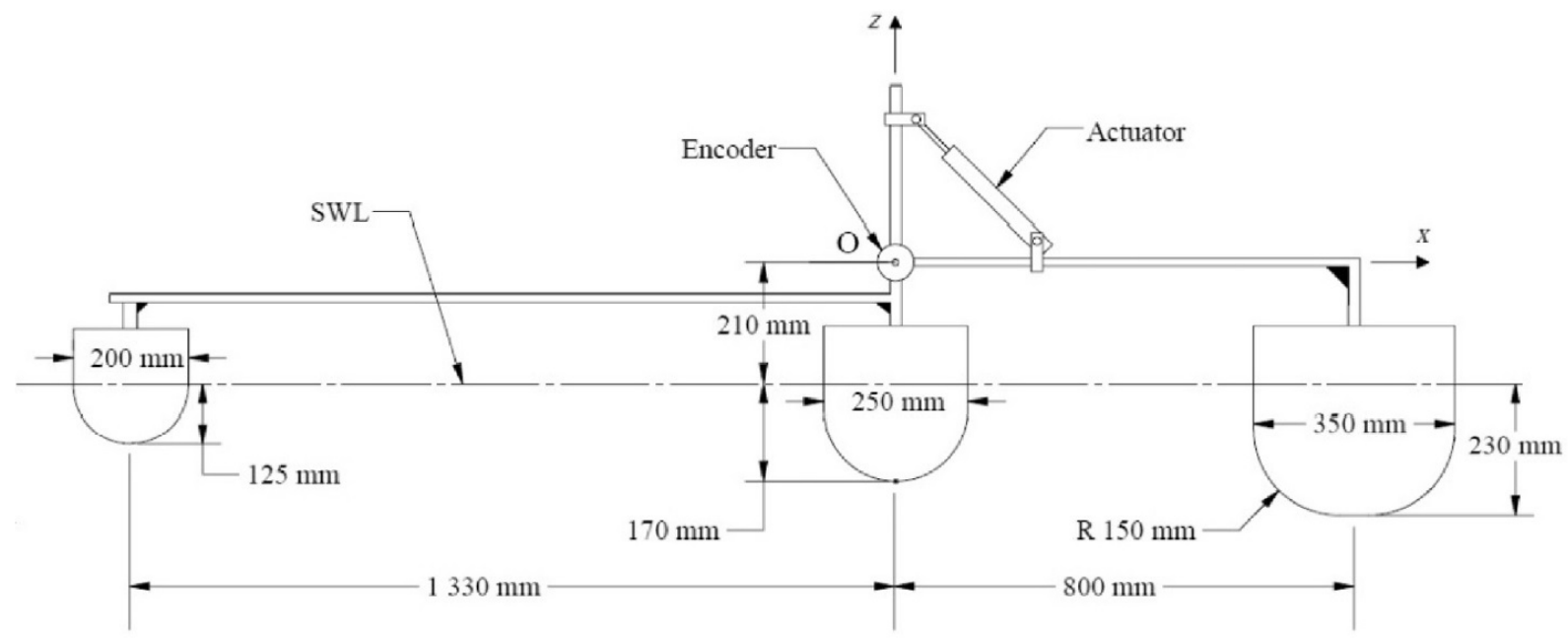

Fig. 1. Diagram of laboratory scale three-float M4 1-1-1 from [2].

2 we introduce the M4 device, and build a control oriented state-space model. In Section 3 the theory of the linear noncausal optimal control is introduced along with some other technical details. Then numerical simulations are demonstrated in Section 4.

\section{THE M4 WEC AND ITS HYDRODYNAMIC MODEL}

\section{A. The M4 WEC}

The M4 wave energy converter was first introduced in [1] as a floating line absorber, and the mechanical design has subsequently been improved [2]. Wave basin experiments were run at different scales to test energy conversion performance and the Froude scaling was confirmed [13]. A linear diffraction model has been built [2] whose fidelity has been validated by tank experiment results with a well-tuned linear damper without control. This provides a good basis for validation of the control-oriented model to be derived. Fig 1 shows the simplest 3-float (1-1-1) format of the M4 device at laboratory scale (approximately 1: 40). The floats are named bow float, mid float and stern float from left to right. 1-1-1 indicates their number. Increasing the number of mid and stern floats will increase the number of power take offs (PTOs) but they are governed by the same operating principles. In this paper only uni-directional waves are considered and the waves are assumed to propagate from left to right in Fig 1 To demonstrate control only the simplest 1-1-1 type is considered, but this may be extended straightforwardly. The 1-1-1 M4 reacts to wave motion with adjacent floats predominantly in anti-phase to generate relative pitch rotation with respect to the hinge at which a PTO is placed to absorb the kinetic energy. The controller is to be designed to control this PTO actively to maximize the energy output.

\section{B. Control-oriented modelling of M4 dynamics}

To design an optimal controller for M4, a state-space model that can describe the M4 dynamics with sufficient accuracy needs to be derived. A controller designed based on a very high order state-space model can result in a prohibitive computational load for real-time implementation. We thus need to develop a state-space model with a sufficiently low order for computational efficiency without sacrificing modelling fidelity significantly. We index the bow float, the mid float, the stern float, the beam connecting the bow and mid float, the beam connecting the mid and stern float, and the power take-off (PTO) with numbers from 1 to 6, respectively. Rotations about the hinge point $O$ in Fig 1 are clockwise positive. Surge, sway, heave, roll, pitch and yaw of a float are the six degrees of freedom (DOF), denoted as modes from 1 to 6, respectively. The working principle determines that M4 only absorbs kinetic energy due to the relative pitch motion while the roll and yaw motions do not contribute. In wave basin experiments, the roll motion of the device is minimised by outrigger buoys either side of the stern float. Yaw motion is not significant since the device aligns naturally with the direction of wave propagation. So it is reasonable to restrict our analysis to the $x-o-z$ plane with modes 1, 3 and 5 only.

1) Motion equation: To derive the equations of the device's motions in the $x-o-z$ plane, a generic and efficient method is to utilize the Euler-Lagrange equation. The notations for the following sections are shown in Table I The generalized coordinate is chosen as $q=\left[x_{0} z_{0} \theta_{1} \theta_{2}\right]^{\top}$, where $\top$ denotes the transpose of a vector. Then the surge motion and the heave motion of each part of the device can be expressed as shown in (11). $\theta$ is assumed to be small, so the approximation $\sin \theta \approx \theta$ holds in the following relations:

$$
\begin{array}{ll}
x_{1}=x_{0}-v_{1} \theta_{1}, & z_{1}=z_{0}+h_{1} \theta_{1} \\
x_{2}=x_{0}-v_{2} \theta_{1}, & z_{2}=z_{0}+h_{2} \theta_{1} \\
x_{3}=x_{0}-v_{3} \theta_{2}, & z_{3}=z_{0}-h_{3} \theta_{2} \\
x_{4}=x_{0}-v_{4} \theta_{1}, & z_{4}=z_{0}+h_{4} \theta_{1} \\
x_{5}=x_{0}-v_{5} \theta_{2}, & z_{5}=z_{0}-h_{5} \theta_{2} \\
x_{6}=x_{0}-v_{6} \theta_{2}, & z_{6}=z_{0}-h_{6} \theta_{2}
\end{array}
$$


TABLE I

NOTATION LIST

\begin{tabular}{ll}
\hline Symbol & Description \\
\hline$i$ & index of each part \\
$r_{i}$ & radius of float $i$ \\
$x_{i}$ & surge motion of $i$ \\
$z_{i}$ & heave motion of $i$ \\
$x_{0}$ & surge motion of hinge $\mathrm{O}$ \\
$z_{0}$ & heave motion of hinge $\mathrm{O}$ \\
$\theta_{1}$ & pitch of $i$ which are on the left of the hinge $\mathrm{O}$ \\
$\theta_{2}$ & pitch of $i$ which are on the right of the hinge $\mathrm{O}$ \\
$q$ & generalized coordinate \\
$h_{i}$ & horizontal distance from COG of $i$ to hinge O \\
$v_{i}$ & vertical distance from COG of $i$ to hinge O \\
$m_{i}$ & mass of $i$, including ballast for floats \\
$I_{i}$ & inertia of $i$ relative to its own COG \\
$\rho$ & water density \\
$g$ & gravitational constant \\
\hline
\end{tabular}

The dynamics of the M4 device can be obtained from the generic Euler-Lagrange equation

$$
\frac{\mathrm{d}}{\mathrm{d} t}\left(\frac{\partial L}{\partial \dot{q}}\right)-\frac{\partial L}{\partial q}=Q
$$

where the Lagrangian $L:=T-V$, with $T$ as the total kinetic energy

$$
\begin{aligned}
T & =\sum_{i=1,2,4}\left[\frac{1}{2} m_{i}\left(\dot{x}_{i}^{2}+\dot{z}_{i}^{2}\right)+\frac{1}{2} I_{i} \dot{\theta}_{1}^{2}\right] \\
& +\sum_{i=3,5,6}\left[\frac{1}{2} m_{i}\left(\dot{x}_{i}^{2}+\dot{z}_{i}^{2}\right)+\frac{1}{2} I_{i} \dot{\theta}_{2}^{2}\right]
\end{aligned}
$$

and $V$ as the total potential energy:

$$
V=\sum_{i=1}^{6} m_{i} g z_{i}
$$

$Q$ is the generalized force acting on the system:

$$
Q=f_{b, q}+f_{w, q}+f_{m o o r, q}+f_{d r a g, q}+f_{p t o, q}
$$

Here $f_{b, q}$ denotes the buoyancy force, which is cancelled by the potential energy derivative term. $f_{w, q}$ denotes the linear wave forces. $f_{\text {moor }, q}$ denotes the mooring force which has a negligible influence on the energy conversion, and is neglected here. $f_{d r a g, q}$ denotes the drag force on the device, which is also negligible for M4 because of its rounded float base design. This is supported by experiment and computational fluid dynamics and drag is reduced further at full scale [14]. $f_{p t o, q}$ denotes the PTO unit torque.

According to the linear diffraction theory, the linear wave force $f_{w, q}$ is composed of the excitation force, the radiation damping force combined with the added mass force and the hydrostatic restoring force [15], and is denoted by

$$
f_{w, q}=f_{e, q}+f_{r d, q}+f_{r s, q}
$$

Excluding these negligible terms from the Lagrangian equation, we derive

$$
M \ddot{q}(t)=f_{e, q}(t)+f_{r d, q}(t)+f_{r s, q}(t)+f_{p t o, q}(t)
$$

Here $M$ comes from the kinetic energy derivative, shown in (8). The diagonal terms of $M$ are due to the summation of the mass and inertia of each float. Non-diagonal terms account for the coupling dynamics between displacements and rotations.

We replace the generalized coordinate index $q$ by $i, j$ to denote the forces or torques acting on float $i$ in mode $j$, with $j=1,3,5$ denoting surge, heave and pitch mode, respectively. Thus, the generalized linear wave forces can be calculated as (9), (10), and (11). Note that all ' $f$ 's are functions of time. Linear wave forces are exerted only on floats. Beams and the PTO unit are above the water surface and have no interaction with waves.

2) Hydrodynamic coefficients and linear wave forces: Hydrodynamic coefficients, derived from a hydrodynamic potential-flow software WAMIT [16], are used to calculate the linear wave forces for each float. The coefficients include excitation force amplitude $F_{e x}$, excitation force phase $\phi$, added mass matrix $A_{\text {inf }}$ for infinite frequency and radiation damping coefficient matrix $B(\omega)$ which provides impulse response functions in the convolution integral as shown below.

Wave excitation force is treated as a disturbance input to the control system. We use the JONSWAP (Joint North Sea Wave Project) wave model with spectral peakedness factor of unity to generate irregular wave spectra with 200 frequency intervals up to maximum of $4 \mathrm{~Hz}$ at model scale which is $3-4$ times of the peak frequency, as for wave profiles used in [2]. Thus, $F_{e x}$ and $\phi$ are matrices of size $200 \times 18$ (here $18=3$ floats $\times 6$ DOFs). The excitation force for float $i$ in mode $j$ is

$$
\begin{array}{r}
f_{e, i, j}(t)=\sum_{n=1}^{200} H(n) F_{e x}(n, 6(i-1)+j) \\
\cos \left(\phi(n, 6(i-1)+j)+\phi_{\text {ran }}(n)\right)
\end{array}
$$

where $H(n)$ and $\phi_{\text {ran }}(n)$ are the amplitude and random phase of JONSWAP wave spectrum, of size $200 \times 1$. Substituting all the ' $f$ ' terms in (9) by (12) yields the final generalized excitation force, which is a $4 \times 1$ vector.

Radiation damping force can be expressed by the Cummins equation [17] which is a convolution of impulse response function (IRF) and the first derivative of a motion. The IRF $L_{m n}$ is calculated by the radiation damping matrix $B_{m n}$ for $m, n=1 \ldots 18$,

$$
L_{m n}(t)=\frac{2}{\pi} \int_{0}^{\infty} B_{m n}(\omega) \cos (\omega t) d \omega
$$

Thus the radiation damping force for float $i$ in mode $j$ in time 


$$
M=\left[\begin{array}{cccc}
\sum_{i=1}^{6} m_{i} & 0 & -m_{1} v_{1}-m_{2} v_{2}-m_{4} v_{4} & -m_{3} v_{3}-m_{5} v_{5}-m_{6} v_{6} \\
0 & \sum_{i=1}^{6} m_{i} & m_{1} h_{1}+m_{2} h_{2}+m_{4} h_{4} & -m_{3} h_{3}-m_{5} h_{5}-m_{6} h_{6} \\
-m_{1} v_{1}-m_{2} v_{2}-m_{4} v_{4} & m_{1} h_{1}+m_{2} h_{2}+m_{4} h_{4} & \sum_{i=1,2,4}\left(I_{i}+m_{i}\left(h_{i}^{2}+v_{i}^{2}\right)\right) & 0 \\
-m_{3} v_{3}-m_{5} v_{5}-m_{6} v_{6} & -m_{3} h_{3}-m_{5} h_{5}-m_{6} h_{6} & 0 & \sum_{i=3,5,6}\left(I_{i}+m_{i}\left(h_{i}^{2}+v_{i}^{2}\right)\right)
\end{array}\right]
$$

$$
\begin{aligned}
& f_{e, q}(t)=\left[\begin{array}{c}
f_{e, 1,1}+f_{e, 2,1}+f_{e, 3,1} \\
f_{e, 1,3}+f_{e, 2,3}+f_{e, 3,3} \\
f_{e, 1,5}+f_{e, 2,5}-f_{e, 1,1} v_{1}-f_{e, 2,1} v_{2}+f_{e, 1,3} h_{1}+f_{e, 2,3} h_{2} \\
f_{e, 3,5}-f_{e, 3,1} v_{3}-f_{e, 3,3} h_{3}
\end{array}\right] \\
& f_{r d, q}(t)=\left[\begin{array}{c}
f_{r d, 1,1}+f_{r d, 2,1}+f_{r d, 3,1} \\
f_{r d, 1,3}+f_{r d, 2,3}+f_{r d, 3,3} \\
f_{r d, 1,5}+f_{r d, 2,5}-f_{r d, 1,1} v_{1}-f_{r d, 2,1} v_{2}+f_{r d, 1,3} h_{1}+f_{r d, 2,3} h_{2} \\
f_{r d, 3,5}-f_{r d, 3,1} v_{3}-f_{r d, 3,3} h_{3}
\end{array}\right] \\
& f_{r s, q}(t)=\left[\begin{array}{c}
f_{r s, 1,1}+f_{r s, 2,1}+f_{r s, 3,1} \\
f_{r s, 1,3}+f_{r s, 2,3}+f_{r s, 3,3} \\
f_{r s, 1,5}+f_{r s, 2,5}-f_{r s, 1,1} v_{1}-f_{r s, 2,1} v_{2}+f_{r s, 1,3} h_{1}+f_{r s, 2,3} h_{2} \\
f_{r s, 3,5}-f_{r s, 3,1} v_{3}-f_{r s, 3,3} h_{3}
\end{array}\right]
\end{aligned}
$$

domain can be calculated as,

$$
\begin{aligned}
f_{r d, i, j}(t) & =\sum_{n=1}^{3} \dot{x}_{n} * L_{6(i-1)+j, 6(n-1)+1} \\
& +\sum_{n=1}^{3} \dot{z}_{n} * L_{6(i-1)+j, 6(n-1)+3} \\
& +\sum_{n=1}^{2} \dot{\theta}_{1} * L_{6(i-1)+j, 6(n-1)+5} \\
& +\dot{\theta}_{2} * L_{6(i-1)+j, 6(n-1)+\left.5\right|_{n=3}}
\end{aligned}
$$

Here the summation index $n$ refers to a float. Notation ' $*$ ' denotes convolution. For example, the portion of radiation damping force acting on float 1 in surge direction caused by the heave motion of float 2 is,

$$
f(t)=\int_{-\infty}^{t} L_{1,9}(t-\tau) \dot{z}_{2}(\tau) d \tau
$$

The lower limit can be set to $t-4 T_{p}$ with sufficient accuracy [2], where $T_{p}$ is the wave peak period. The convolution calculation is time-consuming, and there are 81 convolutions in total to be calculated according to the above analysis. Substituting all ' $f$ 's in (10) by (14) and applying the linear property of convolution and introducing the motions of each float into the generalized variable by (1), we can write the generalized radiation force as

$$
f_{r d, q}(t)=\int_{t-4 T_{p}}^{t} F_{r d}(t-\tau) \dot{q}(\tau) d \tau
$$

where $F_{r d}$ is a $4 \times 4$ matrix with an IRF of length $4 T_{p}$ in each entry. $\dot{q}$ is the first derivative of the generalized coordinate vector. Now the number of convolutions to be calculated is reduced to 16 . A state-space model can be derived from each convolution term, as shown in [18]. The Hankel singular value decomposition algorithm is used to convert each convolution term to a state-space model. The order of the model is proportional to the length of the IRF $F_{r d, m n}$ and can be very high; in this case study, it is around 400. Then assembling the 16 converted state-space models into one statespace model gives an order of around $6400 \times 6400$, which is too high for controller design and real-time implementation of a model-based control algorithm. Thus, model order reduction is necessary for each state-space model. The truncated balanced reduction method is employed to reduce the state-space model for each subsystem corresponding to each convolution term of radiation force from an order of around 400 to a model with an order of 3 to 8 . System identification and the truncated balanced reduction method are implemented using MATLAB routines imp2ss() and balmr(), respectively. A model with an order of 8 for each subsystem is chosen for simulation. Validation is shown in the next section. Now the generalized radiation damping force can be expressed as,

$$
\begin{aligned}
\dot{z}_{s} & =A_{s} z_{s}+B_{s} \dot{q}(t) \\
f_{r d, q}(t) & =C_{s} z_{s}+D_{s} \dot{q}(t)
\end{aligned}
$$

where $z_{s}$ is the state variable of the identified and assembled system with an order of $128 . A_{s}, B_{s}, C_{s}, D_{s}$ are the statespace realisation matrices. Their sizes are $128 \times 128,128 \times 4$, $4 \times 128,4 \times 4$, respectively. The added mass matrix $A_{\text {inf }}$ when the frequency approaches infinity is of size $18 \times 18$, with only a constant value in each entry. The added mass term can be viewed as a force related to the second derivative of the generalized vector variable, $\ddot{q}(t)$. It can also be added to the matrix $M$, after reassembling as a $4 \times 4$ matrix $m_{\infty}$ following the same method for calculating radiation damping force in (10), which is adopted here.

Hydrostatic restoring force is dependent on the heave displacement and pitch rotation, but not on surge in a linear context, i.e. $f_{r s, i, 1}=0$. The heave restoring force for float $i$ is $f_{r s, i, 3}=-\rho g \pi r_{i}^{2} z_{i}$, and the pitch restoring torque for float $i$ is $f_{r s, i, 5}=-\rho g \pi \frac{r_{i}^{4}}{4} \theta_{1 o r 2}$. From (11), the generalized hydrostatic restoring force can be written in a matrix form as

$$
f_{r s, q}(t)=K q(t)
$$


where $K$ is the $4 \times 4$ hydrostatic restoring force matrix

$$
K=\left[\begin{array}{cccc}
0 & 0 & 0 & 0 \\
0 & \sum_{i=1}^{3} k_{z i} & k_{z 1} h_{1}+k_{z 2} h_{2} & -k_{z 3} h_{3} \\
0 & k_{z 1} h_{1}+k_{z 2} h_{2} & \sum_{i=1}^{2} k_{r i}+k_{z i} h_{i}^{2} & 0 \\
0 & -k_{z 3} h_{3} & 0 & k_{r 3}+k_{z 3} h_{3}^{2}
\end{array}\right]
$$

$k_{z i}=-\rho g \pi r_{i}^{2}, k_{r i}=-\rho g \pi \frac{r_{i}^{4}}{4}$.

3) Final state-space representation: To sum up, the motion equation for M4 can be written as

$$
\begin{aligned}
\left(M+m_{\infty}\right) \ddot{q}(t)+f_{r d, q}(t)+K q(t) & =f_{e, q}(t)+f_{p t o, q}(t) \\
\dot{z}_{s} & =A_{s} z_{s}+B_{s} \dot{q}(t) \\
f_{r d, q}(t) & =C_{s} z_{s}+D_{s} \dot{q}(t)
\end{aligned}
$$

When no active control is applied, the PTO is a passive damper, and the PTO torque $M_{m e c h}$ is modelled as $M_{\text {mech }}=$ $-B_{\text {mech }} \dot{\theta}_{r}$, where $B_{\text {mech }}$ is a constant damping ratio and $\dot{\theta}_{r}:=\dot{\theta}_{1}-\dot{\theta}_{2}$ is the relative pitch rotation velocity. When designing a controller, the PTO force is viewed as a manipulatable control input to the WEC system.

By defining a new state vector $x:=\left[\begin{array}{ll}q, \dot{q}, z_{s}\end{array}\right]^{\top}$ and the system output $z=\dot{\theta}_{r}$, the final state-space representation of the control-oriented model for M4 can be written as

$$
\begin{aligned}
\dot{x} & =A x+B_{w} f_{e, q}(t)+B_{u} f_{p t o, q}(t) \\
z & =C x
\end{aligned}
$$

where the system matrices are

$$
\begin{aligned}
& A=\left[\begin{array}{ccc}
0_{4 \times 4} & I_{4 \times 4} & 0_{4 \times n} \\
-\left(M+m_{\infty}\right)^{-1} K & -\left(M+m_{\infty}\right)^{-1} D_{s} & -\left(M+m_{\infty}\right)^{-1} C_{s} \\
0_{n \times 4} & B_{s} & A_{s}
\end{array}\right] \\
& B_{w}=\left[\begin{array}{c}
0_{4 \times 4} \\
\left(M+m_{\infty}\right)^{-1} \\
0_{n \times 4}
\end{array}\right] \\
& B_{u}=\left[\begin{array}{c}
0_{4 \times 1} \\
\left(M+m_{\infty}\right)^{-1}[0,0,1,-1]^{\top} \\
0_{n \times 1}
\end{array}\right] \\
& C=\left[\begin{array}{llll}
0_{1 \times 6} & 1 & -1 & 0_{1 \times n}
\end{array}\right]
\end{aligned}
$$

with $A \in \mathbb{R}^{136 \times 136}$

This results in a single-input-single-output state-space model. The single input is the manipulatable PTO control input $f_{p t o, q}(t)$, and single output is the relative pitch velocity $\dot{\theta}_{r}$. The wave excitation force is treated as a persistent disturbance to the system. The resulting state-space model for M4 is marginally stable. It is also controllable and observable.

\section{LineAR NON-CAUSAl OPTIMAL CONTROLlER DESIGN FOR M4}

\section{A. The control problem formulation}

With the control-oriented model presented in the last section, we can formulate the optimal control problem as follows:

$$
\min _{u_{0}, \ldots, u_{N}} \Sigma_{k=0}^{N}\left\{z_{k} u_{k}+\frac{1}{2} x_{k}^{T} Q x_{k}+\frac{1}{2} r u_{k}^{2}\right\}
$$

subject to the discrete-time model state-space model

$$
\begin{aligned}
x_{k+1} & =A x_{k}+B_{w} w_{k}+B_{u} u_{k} \\
z_{k} & =C x_{k}
\end{aligned}
$$

which is discretized from the continuous time model (21). Here $w_{k}$ is the wave excitation force at time $k$ and $u_{k}$ is the control input at time $k$. The cost function consists of three terms. For the first term, since the power output is $P_{k}=$ $-z_{k} u_{k}$, minimisation of $z_{k} u_{k}$ is equivalent to maximizing the power output. The second term represents the soft constraints on the state vector $x_{k}$. The weight $Q$ is tuned to penalize some motions of M4. The third term aims to penalize the PTO torque by tuning the weight $r$. A good trade-off between these tuning weights needs to be determined to achieve the maximum energy output while not violating the constraints on the PTO limit and M4 motions for its safe operation. $N$ is the number of time steps in the optimization process. In this case it is set to $N \rightarrow \infty$. The weighting matrices $Q$ and $r$ are positive definite. They are tuning parameters to ensure stability of the control system and handle state constraints and input constraints respectively.

\section{B. Non-causal control policy}

The control law [4] derived from resolving the non-causal optimal control problem presented in the last sub-section has the form of

$$
u_{k}=K_{x} x_{k}+K_{d} w_{k, n p}
$$

which consists of a feedback term for the system states $x_{k}$ and a feed-forward term to incorporate the prediction of the incoming wave excitation force $w_{k, n p}:=\left[w_{k}, w_{k+1}, \ldots, w_{k+n_{p}-1}\right]$ where $n_{p}$ is the length of wave prediction horizon. A noncausal controller means the future information contributes to the controller's decision making. In this paper, we assume the incoming wave prediction available. $K_{x}$ and $K_{d}$ are constant coefficient matrices that can be pre-calculated offline. According to [4], the formulae for calculating them are

$$
\begin{aligned}
& K_{x}=-\left(r+B_{u}^{T} V B_{u}\right)^{-1}\left(C+B_{u}^{T} V A\right) \\
& K_{d}=-\left(r+B_{u}^{T} V B_{u}\right)^{-1} B_{u}^{T} \Psi
\end{aligned}
$$

and

$$
\begin{gathered}
V=Q+A^{T} V A-\left(C+B_{u}^{T} V A\right)^{T} \\
\left(r+B_{u}^{T} V B_{u}\right)^{-1}\left(C+B_{u}^{T} V A\right)
\end{gathered}
$$

where $V$ is the algebraic Ricatti equation. Results in [4] show that with an infinite control horizon $N \rightarrow \infty$ and a finite wave prediction horizon $n_{p}$, the control law yields an unique solution.

The only remaining unknown is $\Psi$ which is needed to calculate the feed-forward gain $K_{d}$. Let $\Phi:=\left(A+B_{u} K_{x}\right)^{T}$, then $\Psi:=\left[V B_{w}, \Phi V B_{w}, \ldots, \Phi^{n_{p}-1} V B_{w}\right]$.

\section{State observer design}

Since the optimal controller has a state feedback term, it requires all state information $x_{k}$ to be available, which in our case is not realistic. This is because the states for the radiation damping do not have physical meanings. Thus, a state observer has to be designed to provide the information of these states for the controller.

A standard Kalman state observer is adopted in this case. The Kalman observer has the M4 control input $u_{k}$ and 
output $y_{k}$ from measurement as the observer's inputs, and an estimated state information $\hat{x}_{k}$ as the output. It runs at every time step for the state estimation.

We denote the former input of non-causal controller with observer $\hat{u}_{k-1}$, and the estimated state $\hat{x}_{k-1}$. The Kalman observer algorithm runs as follows: firstly, calculate a priori estimation with the former state information

$$
\hat{x}_{k}^{-}=A \hat{x}_{k-1}+B_{u} \hat{u}_{k-1}+B_{w} w_{k-1}
$$

and then the error covariance $P_{k}^{-}$of this priori estimation is calculated with the predefined model error covariance $Q_{k a l}$

$$
P_{k}^{-}=A P_{k-1} A^{T}+Q_{k a l}
$$

The Kalman observer gain is

$$
K_{k a l}=P_{k}^{-} C^{T}\left(C P_{k}^{-} C^{T}+R_{k a l}\right)^{-1}
$$

where $R_{k a l}$ is the covariance of the measurement. The estimated state information can be calculated as

$$
\hat{x}_{k}=\hat{x}_{k}^{-}+K_{k a l}\left(y_{k}-C x_{k}^{-}\right)
$$

The last step in a Kalman algorithm loop is to update the error covariance matrix $P_{k}$ for the next time step

$$
P_{k}=\left(I-K_{k a l} C\right) P_{k}^{-}
$$

The non-causal optimal control policy with the states estimated by a Kalman observer can be rewritten as

$$
\hat{u}_{k}=K_{x} \hat{x}_{k}+K_{d} w_{k, n p}
$$

In simulation, the measurement of system output $y_{k}$ is set to be the state space model output $z_{k}=y_{k}=C x_{k}$ with added random measurement errors which have a pre-defined error covariance.

The control block diagram is illustrated in Fig. 2 .

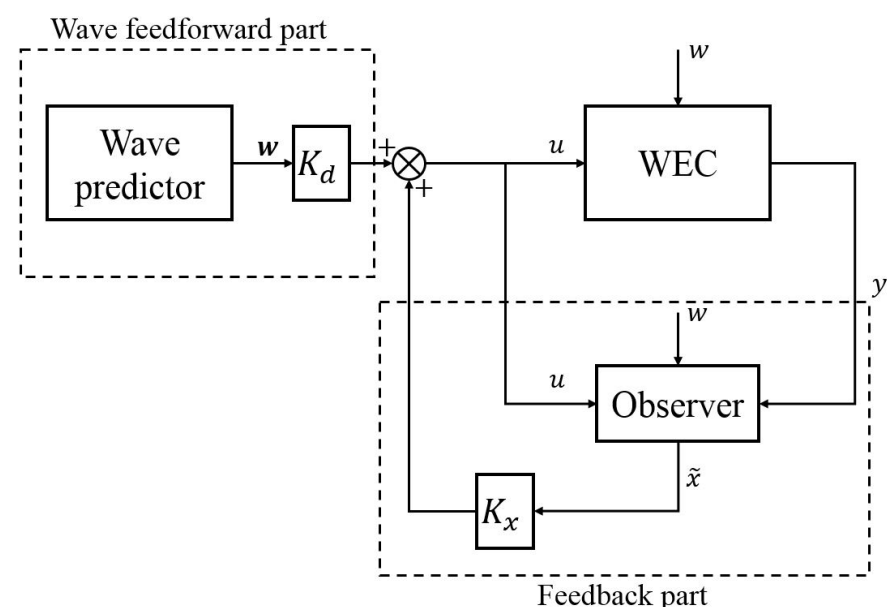

Fig. 2. Linear non-causal optimal control framework [4.

\section{NUMERICAL RESULTS AND DISCUSSION}

Numerical simulations are carried out to compare energy conversion with the designed non-causal optimal controller and a well-tuned passive damper. Simulations are presented in the following order.

Firstly, simulation with a JONSWAP wave profile (tested with $H_{s}=0.04 m, T_{p}=1.8 s$ ) is used to validate the controller performance. Controller is implemented with the pre-tuned parameters $Q, r$ and fixed wave prediction horizon $n_{p}$. Energy, power, control input and relative pitch angle of the device are all plotted to give complete observation and comparison.

Secondly, prediction errors are introduced to demonstrate how robust the controller is to wave prediction errors, and the length of wave prediction $n_{p}$ is varied to show how it affects the controller performance. This is a rather important issue because generally the prediction error increases with the length of the prediction horizon.

Thirdly, simulations of different wave profiles (defined by significant wave height $H_{s}$ and peak period $T_{p}$ ) are run, to compare the controller performance in different sea states. To normalise the average power capture $P_{a v}$ the wave energy propagation per metre crest width $P_{w}$ is used to define capture width as $P_{a v} / P_{w}$. The maximum capture width for point absorbers [19] and hinged rafts [20] in regular waves has been shown theoretically to be proportional to wave length. In the irregular waves of this study we normalise capture width by the wavelength for the energy period to define the capture width ratio which can thus be compared with some reference values. It has also been shown experimentally that the variation of CWR defined in this way with peak period is almost independent of typical spectral peakedness values of 1 and $3.3[9]$. Note the body width has sometimes been used to define capture width ratio.

Finally, a series of controllers with different weighting parameters $Q$ and $r$ are adopted to provide some indications of how to tune the controller to achieve better performance.

\section{A. Discussion of results}

Fig. 3 and Fig. 4 show that with the same wave profile ( $\left.H_{s}=0.04 m, T_{p}=1.8 s\right)$ as in the modelling stage, the noncausal optimal controller with a 3.6 seconds $\left(2 T_{p}\right)$ forward wave excitation force prediction remarkably improves the energy output (at 50 seconds going from $6.06 \mathrm{~J}$ with a welltuned passive damper to $10.89 \mathrm{~J}$ with the controller). The relative pitch angle also has an increase from $1.54^{\circ}$ to $3.74^{\circ}$ RMS value. The input torque maximum amplitude resulting from a control signal is however quite similar to the case when no control is applied. This means the non-causal controller does not require a higher demand on the torque limit of the actuator.

Fig. 5 shows the energy conversion when wave predictions are subject to errors. We introduce two types of errors to the prediction as can be seen in the upper sub-plot of Fig. 5. The black line shows the case when prediction is contaminated by measurement noise represented by White Gaussian Noise (WGN). For the pink line a sequence of noises with ramped magnitudes are added to prediction to enlarge the prediction 


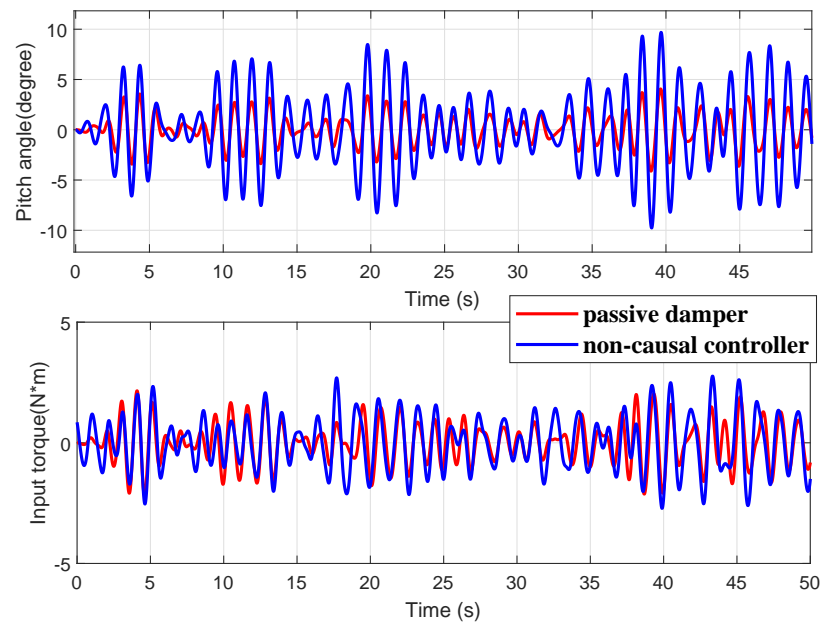

Fig. 3. Relative pitch and input torque, JONSWAP wave $H_{s}=0.04 m$, $T_{p}=1.8 s$, prediction horizon $2 T_{p}=3.6 s\left(n_{p}=400\right)$.
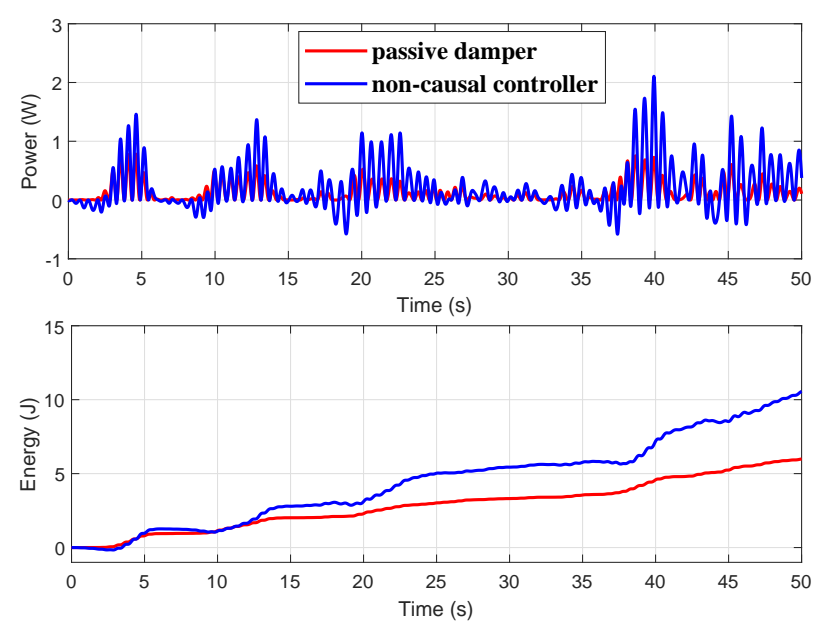

Fig. 4. Power and energy, JONSWAP wave $H_{s}=0.04 m, T_{p}=1.8 \mathrm{~s}$, prediction horizon $2 T_{p}=3.6 s\left(n_{p}=400\right)$.
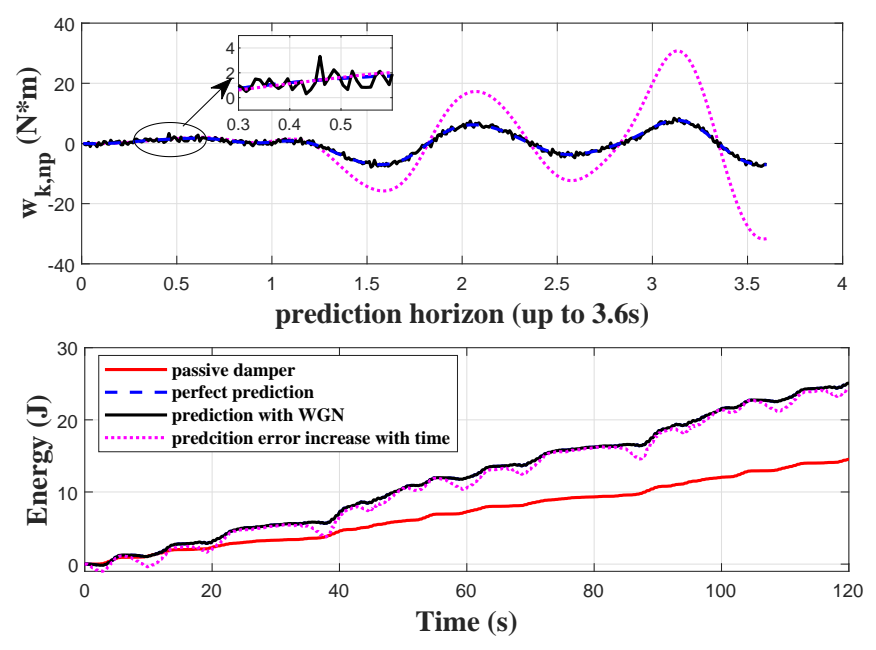

Fig. 5. Energy conversion with prediction error.

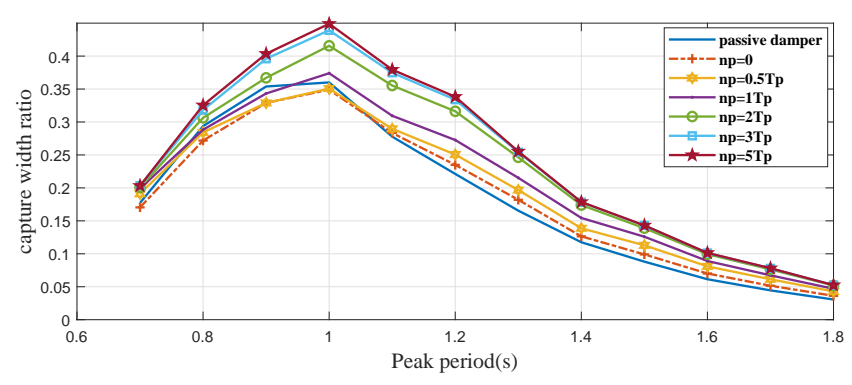

Fig. 6. Capture width ratio with different length of forward wave prediction $n_{p}$
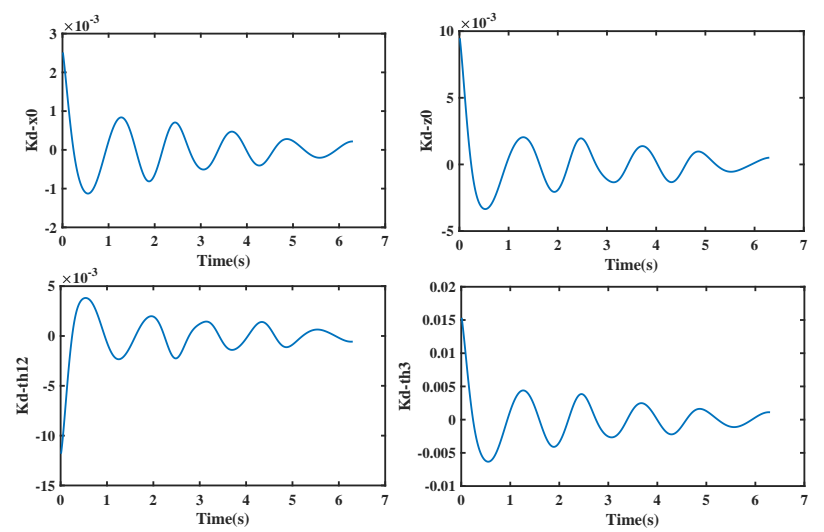

Fig. 7. Feed forward gain $K_{d}$ in surge, heave, pitch mode, as prediction horizon proceeds.

error with time. The reason for doing this is that most of the wave prediction techniques can provide better prediction in the near future than for longer times. Energy conversion of both cases can be seen in the bottom sub-plot of Fig. 5 and compared to the results of Fig. 4. For the WGN case energy conversion is hardly affected. This shows the controller is very robust against this kind of prediction error, which normally occurs with sensors. When the prediction error increases with time, the controller performs slightly worse compared with the WGN case.

Fig. 6 shows the non-causal optimal controller's performance with different prediction horizons. The length of prediction is normalised with the peak wave period $T_{p}$. CWR plot shows that with a longer prediction horizon $n_{p}$, the controller has better performance. However, the influence of the prediction horizon starts to decrease as $n_{p}$ increases to a big enough value. $n_{p}=3 T_{p}$ provides almost optimal control performance, and this length of prediction horizon can be achieved by the DSWP technique [12]. Fig. 7 provides better observation for the feed-forward gain $K_{d}$ which decreases to 0 as the prediction horizon prolongs.

Fig. 8 shows the CWR of the device obtained from a wide range of simulations under JONSWAP wave spectra with $H_{s}=0.035 \mathrm{~m}, H_{s}=0.05 \mathrm{~m}$ and $H_{s}=0.07 \mathrm{~m}$. Wave peak period ranges from $0.7 s$ to $1.8 s$ with $0.1 s$ interval. Results with a passive damper (dashed line) are close to CWR obtained in a tank experiment [2], validating the modelling fidelity. With our non-causal controller (solid line), the CWR of the device 


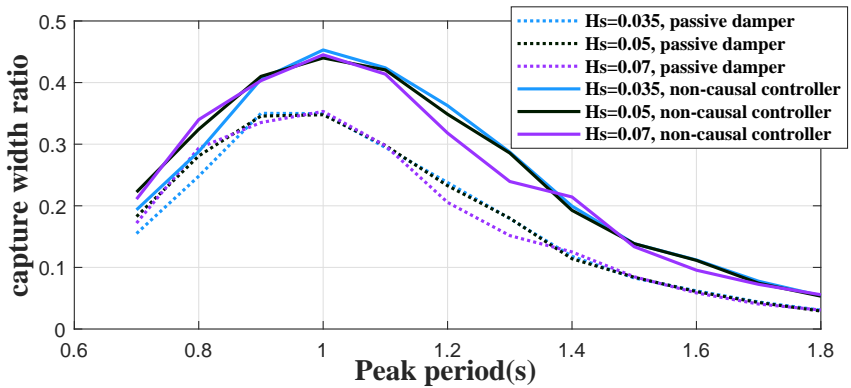

Fig. 8. Capture width ratio in different wave height and peak period, prediction horizon $5 T_{p}$.

improves across a wide range of peak periods. Within peak wave periods of 1-1.8 seconds tank scale (6-11 seconds in full scale), the improvement changes from $40 \%$ at wave peak period $T_{p}=1 \mathrm{~s}$ to $100 \%$ at wave peak period $T_{p}=1.8 \mathrm{~s}$. Note that the same controller parameters $Q$ and $r$ are used for different wave conditions (significant heights and peak periods), and the results show that tuning of $Q$ and $r$ is not sensitive to wave conditions. This will ease the controller implementation efforts in real applications. It can also be seen that CWR is almost independent of the significant wave height and is determined by the wave peak periods as expected with this form of non-dimensional CWR.

Figs. 9 $910 \mid 11$ provide more insights on how to tune the controller weighting matrices $Q$ and $r$. Simulations are run using JONSWAP waves with $H_{s}=0.035 \mathrm{~m}$, and a wave prediction horizon of 3.6 seconds $\left(n_{p}=400\right)$. The red line is the CWR of the device with a passive damper for comparison purpose. The states weighting matrix $Q$ is divided into two parts which are tuned separately. The first part is for the first eight states which are the displacement and velocity of the device, denoted by $q_{1}$. The second part is for the states without any physical meaning corresponding to the radiation subsystems, denoted by $q_{2}$. The structure of $Q$ is in the form of

$$
Q=\left[\begin{array}{cc}
q_{1} I_{8} & 0 \\
0 & q_{2} I_{n}
\end{array}\right]
$$

with $I_{n}$ denoting an $n$ by $n$ identity matrix. Control input weighting is denoted by $r$. Note that tuning of parameter $r$ should be considered together with the control actuator torque limit since a smaller value of $r$ can lead to larger control input magnitude. $r=0.08$ is chosen for simulations carried out in this paper after the tuning procedure.

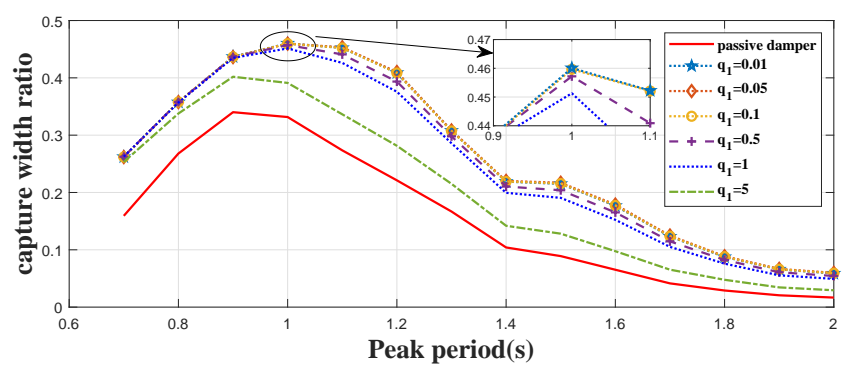

Fig. 9. Tuning of controller parameter $Q$, for states with physical meanings.

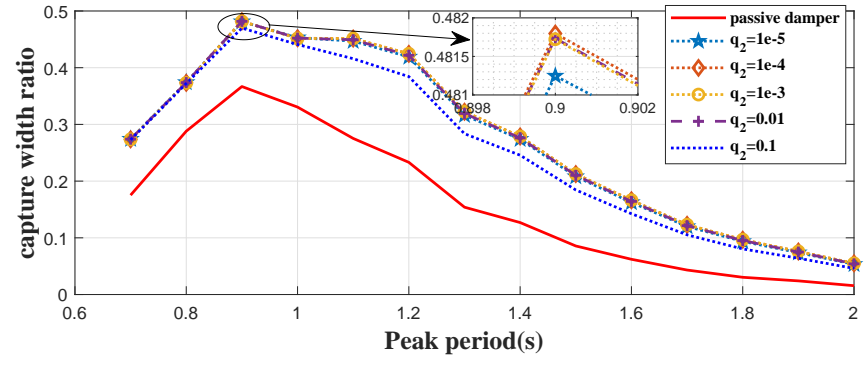

Fig. 10. Tuning of controller parameter $Q$, for states without physical meanings.

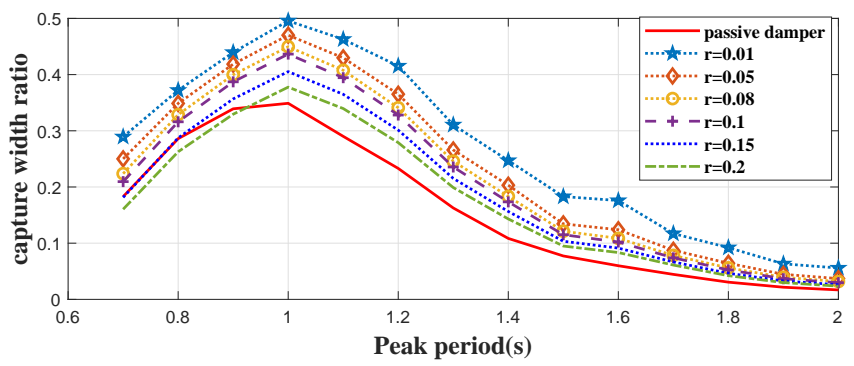

Fig. 11. Tuning of controller parameter $r$.

\section{B. Implications}

These improvements in CWR require mechanical PTO hardware and power electronics to be available but this is an area of active development, notably for wind turbines. For wave energy estimates of annual electricity generation for various worldwide sites have been made without control for configurations with 3 to 8 floats [2], [9]. Electricity cost estimates were also made following standard discounting practices. The basic 3 -float (1-1-1) system investigated here provides an annual average power at Wave Hub in Cornwall of $280 \mathrm{~kW}$ and for the 6-float (1-3-2) system with two PTOs the annual average becomes $660 \mathrm{~kW}$. For Belmullet in W Ireland a somewhat larger scale system is desirable to minimise electricity cost giving an average power for the 1-1-1 system of $970 \mathrm{~kW}$ and $2290 \mathrm{~kW}$ for the 1-3-2 system. This gives capacities similar to offshore wind turbines and control will increase energy yield and hence capacities substantially. Since control is relatively inexpensive electricity cost will almost certainly be reduced although this is hard to quantify as hardware implications are not known. An important consideration is that the improvement in CWR by control increases as wave period increases. This means that the device size required to minimise electricity cost will be smaller with control than without, thereby reducing the overall cost which is proportional to scale to a power between 2 and 3 . Accurate costing obviously requires detailed engineering design of all aspects.

\section{CONCLUSION}

A linear non-causal optimal controller is designed for M4, a reconfigurable multi-float and multi-mode-motion wave energy converter. The simplest 3-float version considered here can be straightforwardly generalised to a larger number of floats with multiple PTOs. The dynamics of M4 can be described 
by a reduced-order linear model with sufficient fidelity, which enables the design and implementation of the linear non-causal optimal controller. Numerical simulations show that the controller with a limited horizon of wave prediction incorporated can significantly improve the capture width by between $40 \%$ and $100 \%$ over a broad range of sea states. Exact improvement in cost of electricity requires detailed engineering design of all aspects.

\section{ACKNOWLEDGEMENT}

Zhijing Liao and Nian Gai are grateful to the financial support from the joint "Queen Mary University of London - China Scholarship Council” scholarship.

This work was also supported in part by a research contract from Wave Energy Scotland's Control Systems programme and in part by the Newton Advanced Fellowship (No. NA160436) from Royal Society.

\section{REFERENCES}

[1] P. Stansby, E. Carpintero Moreno, T. Stallard, and A. Maggi, "Three-float broad-band resonant line absorber with surge for wave energy conversion," Renewable Energy, vol. 78, pp. 132 - 140, 2015. [Online]. Available: http://www.sciencedirect.com/science/article/ pii/S0960148114008957

[2] P. Stansby, E. Carpintero Moreno, and T. Stallard, "Large capacity multi-float configurations for the wave energy converter $\mathrm{m} 4$ using a time-domain linear diffraction model," Applied Ocean Research, vol. 68, pp. 53 - 64, 2017. [Online]. Available: http://www.sciencedirect.com/ science/article/pii/S0141118717302146

[3] A. F. de O. Falcão, "Wave energy utilization: A review of the technologies," Renewable and Sustainable Energy Reviews, vol. 14, no. 3, pp. 899 - 918, 2010. [Online]. Available: http://www.sciencedirect.com/science/article/pii/S1364032109002652

[4] S. Zhan and G. Li, "Linear noncausal optimal control of wave energy converters," IEEE Transactions on Control System Technology, 2018.

[5] G. Li and M. R. Belmont, "Model predictive control of sea wave energy converters-part i: A convex approach for the case of a single device," Renewable Energy, vol. 69, pp. 453-463, 2014.

[6] S. Zhan, W. He, and G. Li, "Robust feedback model predictive control of sea wave energy converters," IFAC-PapersOnLine, vol. 50, no. 1 , pp. 141 - 146, 2017, 20th IFAC World Congress. [Online]. Available: http://www.sciencedirect.com/science/article/pii/S2405896317300368

[7] G. Li, "Nonlinear model predictive control of a wave energy converter based on differential flatness parameterisation," International Journal of Control, vol. 90, no. 1, pp. 68-77, 2017.

[8] R. Genest and J. V. Ringwood, "A critical comparison of modelpredictive and pseudospectral control for wave energy devices," Journal of Ocean Engineering and Marine Energy, vol. 2, no. 4, pp. 485-499, 2016.

[9] E. Carpintero Moreno and P. Stansby, "The 6-float wave energy converter M4: ocean basin tests giving capture width, response and energy yield for several sites," Renewable and Sustainable Energy Reviews, vol. 104, pp. $307-318,2019$.

[10] R. Eatock Taylor, P. Taylor, and P. Stansby, "A coupled hydrodynamic-structural model of the M4 wave energy converter," Journal of Fluids and Structures, vol. 63, pp. 77 - 96, 2016. [Online]. Available: http://www.sciencedirect.com/science/article/pii/S0889974616000372

[11] L. Sun, J. Zang, P. Stansby, E. C. Moreno, P. H. Taylor, and R. E. Taylor, "Linear diffraction analysis of the three-float multi-mode wave energy converter M4 for power capture and structural analysis in irregular waves with experimental validation," Journal of Ocean Engineering and Marine Energy, vol. 3, no. 1, pp. 51-68, Feb 2017. [Online]. Available: https://doi.org/10.1007/s40722-016-0071-5

[12] L. Abusedra and M. Belmont, "Prediction diagrams for deterministic sea wave prediction and the introduction of the data extension prediction method," vol. 58, pp. 59-81, 012011.
[13] P. Stansby, E. Carpintero Moreno, and T. Stallard, "Capture width of the three-float multi-mode multi-resonance broadband wave energy line absorber M4 from laboratory studies with irregular waves of different spectral shape and directional spread," Journal of Ocean Engineering and Marine Energy, vol. 1, no. 3, pp. 287-298, Aug 2015. [Online] Available: https://doi.org/10.1007/s40722-015-0022-6

[14] H. Gu, P. Stansby, T. Stallard, and E. Carpintero Moreno, "Drag, added mass and radiation damping of oscillating vertical cylindrical bodies in heave and surge in still water," Journal of Fluids and Structures, vol. 82, p. 343-356, 102018.

[15] Z. Yu and J. Falnes, "State-space modelling of a vertical cylinder in heave," Applied Ocean Research, vol. 17, no. 5, pp. 265 - 275, 1995. [Online]. Available: http://www.sciencedirect.com/science/article/ pii/0141118796000028

[16] C. Lee and J. N. Newman, "Wamit - user manual version 7.0," in WAMIT Inc, Chestnut Hill, Massachusetts, 2013.

[17] W.E.Cummins, "The impulse response function and ship motions." [Online]. Available: https://dome.mit.edu/handle/1721.3/49049

[18] E. Kristiansen, Åsmund Hjulstad, and O. Egeland, "State-space representation of radiation forces in time-domain vessel models," Ocean Engineering, vol. 32, no. 17, pp. 2195 - 2216, 2005. [Online]. Available: http://www.sciencedirect.com/science/article/pii/S0029801805000946

[19] J. Falnes, Ocean Waves and Oscillating Systems: Linear Interactions Including Wave-Energy Extraction. Cambridge University Press, 2002.

[20] J. Newman, "Absorption of wave energy by elongated bodies," Applied Ocean Research, vol. 1, no. 4, pp. 189 - 196, 1979. [Online]. Available: http://www.sciencedirect.com/science/article/pii/0141118779900269

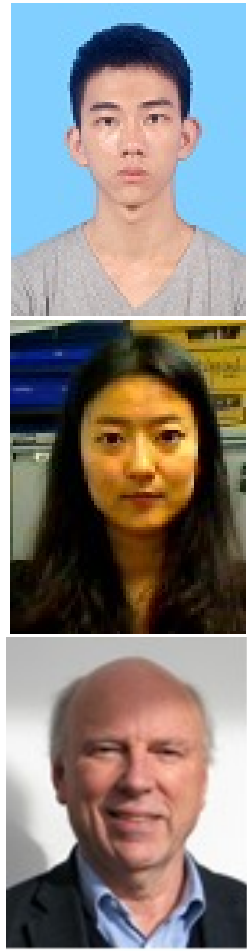

Zhijing Liao was born in Guangdong China in 1994. He received his double bachelor degree from Beijing Institute of Technology, Beijing, China, and Polytechnic University of Turin, Turin, Italy, in 2017. $\mathrm{He}$ is currently working toward the Ph.D. degree at Queen Mary University of London, London, U.K. His research interests include modelling and control of wave energy converters.

Nian Gai is a PhD student in the school of engineering and material science in the Queen Mary, University of London. Her research topic is on optimal control and co-design problem for wave energy converters.

Peter Stansby is the inaugural Osborne Reynolds Professor of Fluid Mechanics in the School of Mechanical, Aerospace and Civil Engineering (MACE) in the University of Manchester and Fellow of the Royal Academy of Engineering. In a long career to date spanning 40 years, he has been a Dynamicist at Atkins Research and Development, a member of the W.S. Atkins Group, a Lecturer, Senior Lecturer, Professor of Hydrodynamics at the Engineering Department of the Victoria University of Manchester, Head of the Manchester Centre for Civil and Construction Engineering, UMIST, Professor of Hydrodynamics in MACE and Head of the School of MACE. Of relevance to this paper he has been developing wave energy devices for a decade through experiments and linear wave/body modelling resulting in the patented multi-float modular system M4.

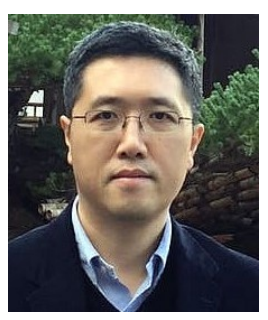

Guang Li (M'09) received his Ph.D. degree in Electrical and Electronics Engineering, specialized in control systems, from the University of Manchester, in 2007 . He is currently a Senior Lecturer in dynamics modelling and control in Queen Mary University of London, UK. His current research interests include constrained optimal control, model predictive control, adaptive robust control and control applications including renewable energies and energy storage, etc. 